\title{
Reverse pseudohyperkalemia in a newly diagnosed pediatric patient with acute T-cell leukemia and hyperleukocytosis: a case report and literature review
}

\author{
Muneera Alabdulqader ${ }^{1}$, Hanaa Al Alawyat ${ }^{3^{*}}$ (D), Mohammed F. Kasem², Abdelfattah Ibrahim ${ }^{3}$ and
} Abdelwahab Omara ${ }^{3}$

\begin{abstract}
Background: Hyperkalemia is a serious medical condition that requires immediate intervention. However, pseudohyperkalemia and reverse pseudohyperkalemia are misleading clinical manifestations that can result in incorrect diagnosis and consequent harmful intervention.

Case presentation: An 11-year-old girl manifested an incidental finding of hyperleukocytosis (WBC $\left.>400 \times 10^{9} / \mathrm{L}\right)$, with $90 \%$ blast cells during routine pre-operative investigations for adenotonsillectomy. Initial investigations demonstrated elevated serum potassium levels $(7.5 \mathrm{mmol} / \mathrm{L})$, despite concomitantly normal levels in venous blood gas samples (3.9-4.4 mmol/L) and being clinically stable with normal 12-lead ECG. Surprisingly, plasma potassium level was exacerbated, in comparison to the serum level by $>1 \mathrm{mmol} / \mathrm{L}$. This finding is consistent with reverse pseudohyperkalemia that is associated with hyperleukocytosis in acute leukemia that does not require any active intervention.

Conclusion: This case report emphasizes the significance of interpreting potassium levels accurately, preferably utilizing whole-blood potassium level over serum and plasma level in newly diagnosed leukemia cases with hyperleukocytosis. Additionally, having a high index for the possibility of reverse pseudohyperkalemia, secondary to leakage from fragile leukocytes, avoids unnecessary treatment that might cause harm to the patient.
\end{abstract}

Keywords: Hyperkalemia, Pseudohyperkalemia, Hyperleukocytosis, Leukemia

\section{Introduction}

Hyperkalemia is a potentially life-threatening condition that requires immediate medical intervention. However, pseudohyperkalemia and reverse hyperkalemia are misleading clinical manifestations that might result in inappropriate patient management. Pseudohyperkalemia (PHK) has been defined as a false increase in serum

\footnotetext{
*Correspondence: hanaa.alawyat@kfsh.med.sa; hanaaalalawyat@gmail.com

${ }^{3}$ Department of Critical Care, Division of Pediatric Critical Care, King

Fahad Specialist Hospital, Ammar Bin Thabit Street, Dammam 32253, Kingdom of Saudi Arabia

Full list of author information is available at the end of the article
}

potassium level, in comparison to a normal plasma level of $\geq 0.4 \mathrm{mmol} / \mathrm{L}$ [1]. This is commonly encountered within settings of high leukocyte or platelet-counts, secondary to the release of intracellular potassium during the process of specimen collection and clot formation [2-6]. Singh and colleagues first-described reverse pseudohyperkalemia (rPHK), wherein plasma potassium concentrations were exacerbated in comparison to serum potassium levels. This study proposed that the underlying mechanism was an increased sensitivity to heparin-induced membrane damage during cases of hematological malignancy [1]. 
Identifying PHK and rPHK in patients with leukemia and hyperleukocytosis carries a significant clinical implication in patient monitoring and management, by avoiding unnecessary interventions that might actually cause harm to such patients. This case report discusses a child with acute leukemia that presented with hyperleukocytosis and hyperkalemia. Such a case emphasized the significance of differentiation between true hyperkalemia, $\mathrm{PHK}$, and rPHK in guiding the appropriate patient management and addressing all possible causes for such clinical manifestations.

\section{Patient and methods}

Previously reported cases were identified by performing a MEDLINE and PubMed review of clinical literature, using the keywords 'reverse pseudohyperkalemia' and'leukemia' (English language) for the time frame of 1964-2020. Any case report with tumor lysis syndrome or true hyperkalemia (secondary to another cause, other than acute leukemia) was excluded, together with animal studies (Table 1). This study identified 14 reported cases, which were similar to the case described in this article (Tables 1,2).

\section{Case report}

An 11-year-old girl with unremarkable medical history was incidentally found to manifest a white blood cell-count (WBC $>400 \times 10^{9} / \mathrm{L}$ ) with $90 \%$ blasts and hyperkalemia, during pre-operative investigations for adenotonsillectomy. The patient was referred to pediatric oncology in our hospital for further management. Initial assessment within the pediatric intensive care unit (PICU) revealed a clinically and hemodynamically stable patient with tonsillar hypertrophy, multiple enlarged cervical lymph nodes, and hepatosplenomegaly. Complete blood count (CBC) of the patient revealed WBC of $440 \times 10^{9} / \mathrm{L}$ with $92 \%$ blasts, hemoglobin count of $(\mathrm{Hgb}) 8.3 \mathrm{~g} / \mathrm{dL}$, and a platelet count $90 \times 10^{9} / \mathrm{L}$. Her initial biochemical (serum) profile revealed a potassium level of $7.5 \mathrm{mmol} / \mathrm{L}$, sodium level of $139 \mathrm{mmol} / \mathrm{L}$, phosphate level of $1.27 \mathrm{mmol} / \mathrm{L}$, calcium level of $2.4 \mathrm{mmol} / \mathrm{L}$, uric acid level of $0.01 \mathrm{mg} /$ $\mathrm{dL}$, lactate dehydrogenase (LDH) level of $1434 \mathrm{u} / \mathrm{L}$, creatinine level of $0.53 \mathrm{mg} / \mathrm{dL}$, together with a blood urea nitrogen level of $4.48 \mathrm{mg} / \mathrm{dL}$. In addition, venous blood gas sample revealed a potassium level of $3.9 \mathrm{mmol} / \mathrm{L}$. There was a noticeable discrepancy between potassium levels in serum and venous blood gas samples (7.5 $\mathrm{mmol} / \mathrm{L}$ and $3.9 \mathrm{mmol} / \mathrm{L}$, respectively). However, the presence of an equivocal, prominent $\mathrm{T}$ wave in the 12-lead Electrocardiography (ECG) investigation triggered the medical team to manage this case as true hyperkalemia, consequently considering the deployment of calcium chloride, sodium bicarbonate bolus infusions and urgent dialysis. Following medical treatment, serum potassium level was $6.8 \mathrm{mmol} / \mathrm{L}$, while the venous blood gas sample exhibited hypokalemia, with a potassium level of $2.5 \mathrm{mmol} / \mathrm{L}$. Serum specimens were transported by pneumatic tube, and the time from phlebotomy to analysis was approximately $30 \mathrm{~min}$ using [Abbott Machine] following a five-minute centrifugation step. No hemolysis was observed, while a whole blood samples was collected in a blood-gas syringe (Q-cork, with $25-\mathrm{u}$ balanced heparin) and immediately analyzed through a bloodgas analyzer machine [GEM premium $4000^{\circledR}$ ] that is located within PICU.

The patient exhibited no acidosis, renal failure, or tumor lysis syndrome. Phosphate, calcium, and uric acid levels were not increased. Consequently, the patient was started on maintenance and a half IV fluid, allopurinol and dexamethasone since admission, awaiting the bone marrow biopsy result.

The wide discrepancy between serum potassium and blood-gas potassium levels raised the possibility of PHK (Table 3, Fig. 1). Additionally, the presence of hyperleukocytosis in the absence of other biochemical markers for tumor lysis in an asymptomatic and clinically stable patient with repeated normal 12 lead ECG and sinus rhythm-supported the possibility of PHK and was consequently investigated accordingly.

Additional venous blood samples were simultaneously obtained at $1 \mathrm{~h}$ post-potassium-lowering treatment (Table 3). These samples were heparin-containing-tube plasma sample, heparin-lacking-tube serum sample, and whole-blood-gas syringe sample. Plasma and serum specimens were immediately transported to the laboratory by hand ( $<5$ min transport time), promptly centrifuged, and analyzed within five minutes of centrifugation, while the whole-blood sample was analyzed immediately through a blood-gas analyzer machine in PICU. Surprisingly, the plasma level demonstrated an exacerbated potassium level of $7.4 \mathrm{mmol} / \mathrm{L}$, in comparison to the serum potassium level $(6.3 \mathrm{mmol} / \mathrm{L})$ and whole-blood potassium level of $2.5 \mathrm{mmol} / \mathrm{L}$ in the blood-gas sample (Table 3 ).

Conclusively, the medical team deemed the high serum potassium level as a rPHK secondary to hyperleukocytosis. Meanwhile, the patient was monitored closely with a continuous cardiac monitor and serial 12-lead ECG readings, along with potassium level investigations from venous blood gas samples every 3-6 h, with no dialysis performed (Fig. 1). Consequently, the medical team decided not to actively intervene unless the patient became symptomatic, or her ECG demonstrated the characteristic changes of hyperkalemia. Fortunately, the patient remained asymptomatic, with normal serial 


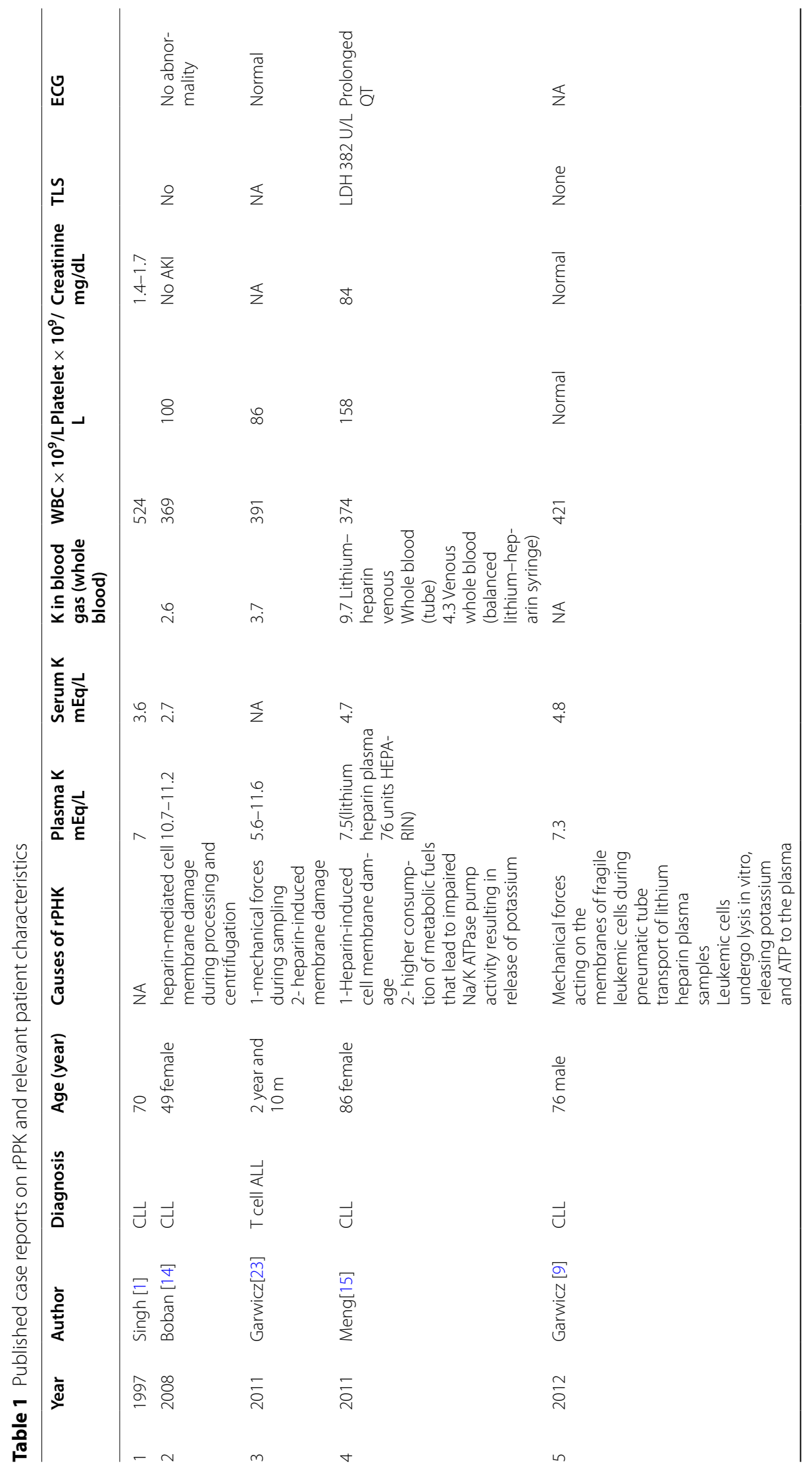




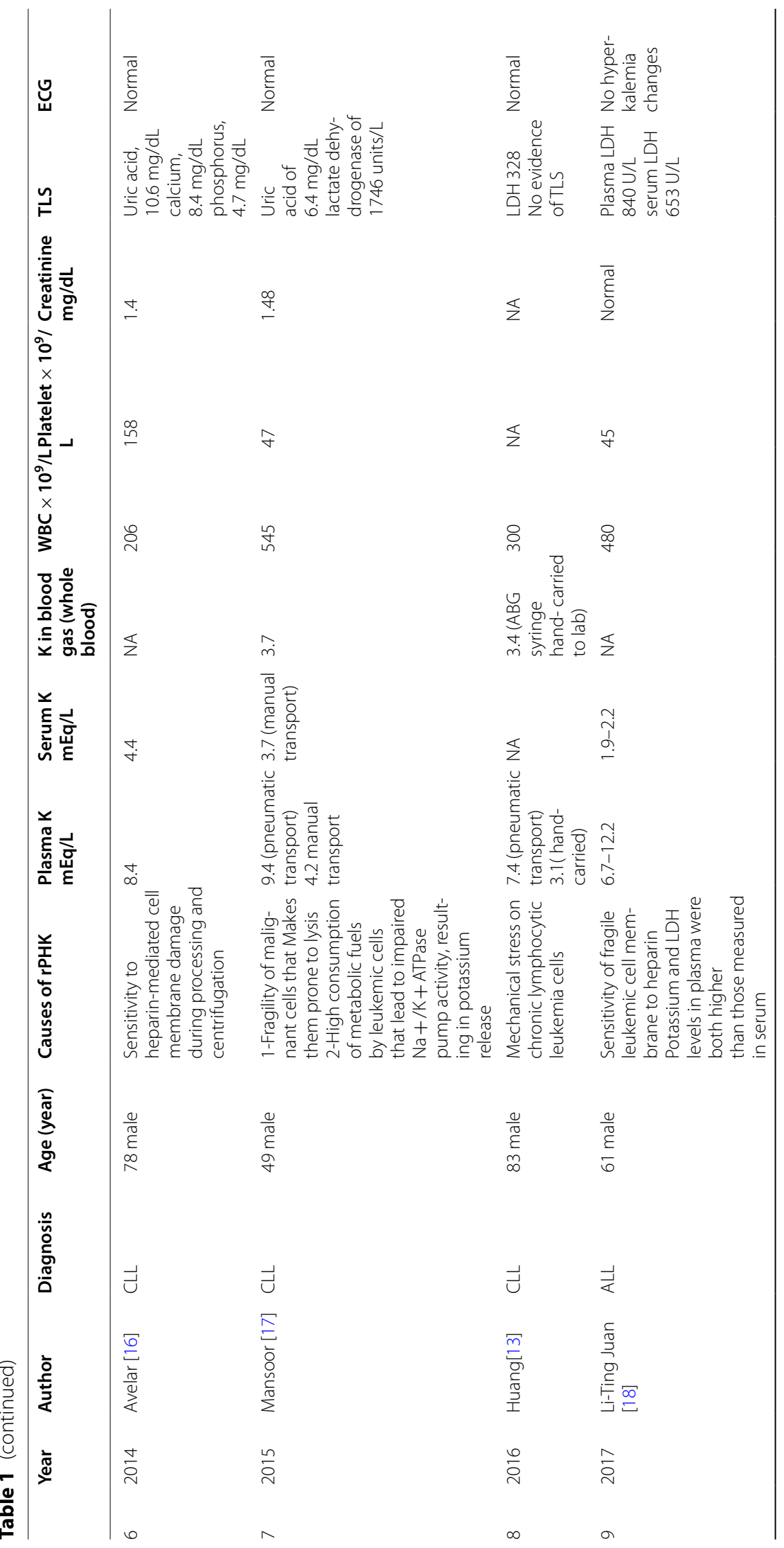




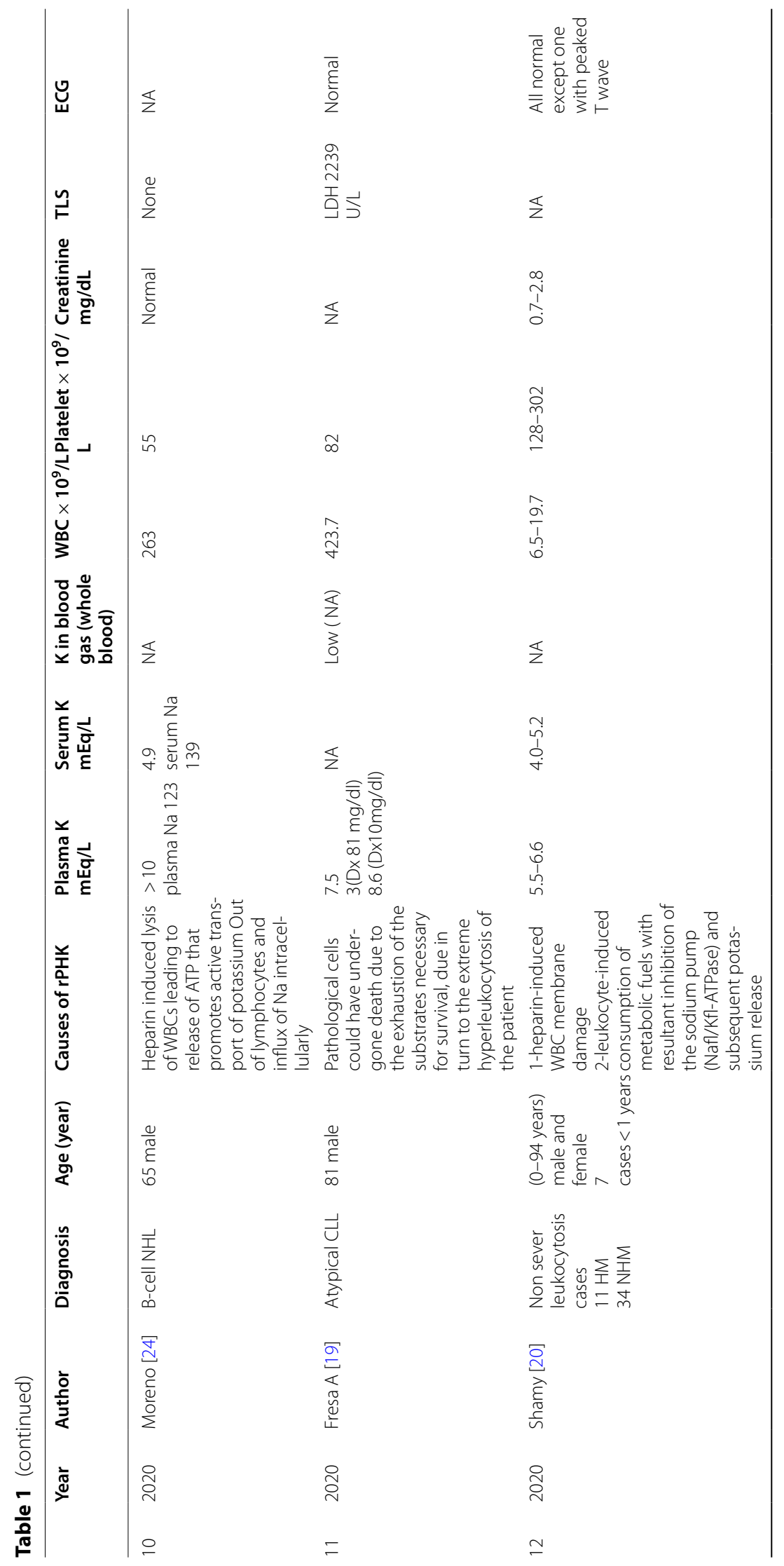




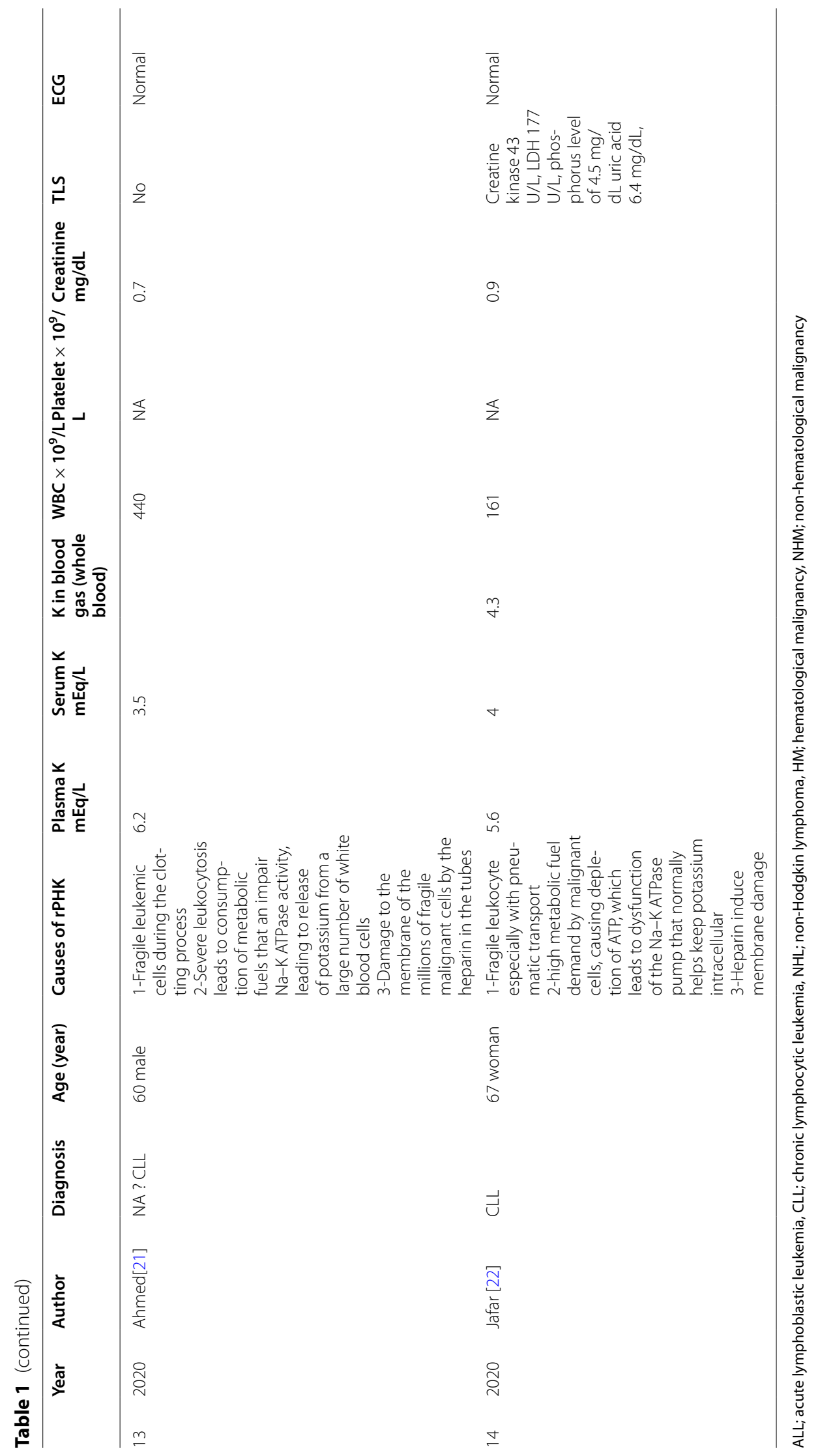


Table 2 Published case reports on rPPK management and outcomes

\begin{tabular}{|c|c|c|c|c|c|}
\hline & Medication used to lower $\mathrm{k}$ & CRRT & $\begin{array}{l}\text { WBC after } \\
\text { chemotherapy }\end{array}$ & $\begin{array}{l}\text { K level after } \\
\text { normalization of WBC } \\
\text { (mmol/L) }\end{array}$ & Outcome \\
\hline 1 & NA & NA & NA & NA & NA \\
\hline 2 & $\begin{array}{l}\text { Calcium chloride, albuterol, dextrose- } \\
\text { insulin, furosemide, and sodium } \\
\text { polystyrene } \\
\text { sulfonate }\end{array}$ & - Planned but not done & NA & NA & $\begin{array}{l}\text { no dialysis was performed, and } \\
\text { potassium } \\
\text { supplements were administered }\end{array}$ \\
\hline 3 & NA & NA & 3.7 & 4 & NA \\
\hline 4 & None & None & NA & NA & NA \\
\hline 5 & None & None & 150 & 4 & \\
\hline 6 & $\begin{array}{l}\text { Intravenous calcium gluconate, } \\
\text { intravenous insulin, and oral sodium } \\
\text { polystyrene sulfonate }\end{array}$ & Partial hemodialysis & NA & NA & $\begin{array}{l}\text { Hemodialysis stoped and patient } \\
\text { treatend as rPHK }\end{array}$ \\
\hline 7 & None & None & NA & 4.3 & $\begin{array}{l}\text { Received chemo without com- } \\
\text { plication and discharge home in } \\
\text { good condition }\end{array}$ \\
\hline 8 & $\begin{array}{l}\text { Insulin plus glucose and sodium } \\
\text { polystyrene }\end{array}$ & NA & NA & $\begin{array}{l}3.1 \text { (after chemo- } \\
\text { therapy) }\end{array}$ & $\begin{array}{l}\text { Received chemotherapy, no TLS } \\
\text { or complication }\end{array}$ \\
\hline 9 & $\begin{array}{l}\text { Insulin and oral sodium } \\
\text { Polystyrene sulfonate }\end{array}$ & Hemodialysis Day 3-6 & 100 & $3-3.2$ & $\begin{array}{l}\text { Chemotherapy initiated } \\
\text { His serum potassium level was } \\
\text { maintained } \\
\text { within the normal range during } \\
\text { the rest of his hospital } \\
\text { stay, whereas WBC counts contin- } \\
\text { ued to decrease } \\
\text { with the progression of chemo- } \\
\text { therapy }\end{array}$ \\
\hline 10 & NA & NA & 364 & WBC Never normalized & $\begin{array}{l}\text { entered palliative care following } \\
\text { multifocal intracerebral hemor- } \\
\text { rhage and died approximately } \\
\text { two weeks after presentation }\end{array}$ \\
\hline 11 & NA & NA & NA & NA & $\begin{array}{l}\text { Paroxysmal atrial fibrillation } \\
\text { (Ibrutinib was suspended and } \\
\text { cardioversion with amiodarone } \\
\text { was performed with success) } \\
\text { Oral Cyclophosphamide with } \\
\text { no SE }\end{array}$ \\
\hline 12 & $\begin{array}{l}17 \text { cases received either SPS, insulin, } \\
\text { furosemide, NaHco3, }\end{array}$ & $\begin{array}{l}\text { Hemodialysis in } 3 \text { cases } \\
\text { (who are CKD or ESRD) }\end{array}$ & NA & NA & $\begin{array}{l}6(15 \%) \text { died during hospitaliza- } \\
\text { tion } 4 \text { received } \\
\text { Potassium-lowering therapy and } \\
4 \text { had an HM }\end{array}$ \\
\hline 13 & NA & NA & NA & NA & NA \\
\hline 14 & $\begin{array}{l}\text { Calcium gluconate, insulin, and dex- } \\
\text { trose cocktail }\end{array}$ & None & & & $\begin{array}{l}\text { Discharged to home and advised } \\
\text { regular follow-ups with her } \\
\text { oncologist }\end{array}$ \\
\hline
\end{tabular}

12-lead ECG readings and normal serial readings of potassium level from venous blood-gas samples (Table 3, Fig. 1).

The following day, the patient was confirmed to have T-cell acute lymphoblastic leukemia and received her first cycle of chemotherapy course while maintaining a normal potassium level (venous blood-gas samples) with no evidence of tumor lysis. Following a further 24-h period, her leukocyte count dropped to $<150 \times 10^{9} / \mathrm{L}$. In addition, serum potassium level normalized to $4.5 \mathrm{mmol} / \mathrm{L}$, matching the venous blood gas sample level (Table 3, Fig. 1).

\section{Discussion}

Hyperkalemia is not uncommon occurrence in pediatric hospital admissions, accounting for up to $29 \%$ of all pediatric intensive care unit admissions [6]. Hyperkalemia is a serious clinical manifestation that requires immediate and effective treatment, since a serum (or plasma) 
Table 3 Serum potassium levels versus blood gas potassium level in relation to WBC counts

\begin{tabular}{lllll}
\hline Sample & Serum Potassium $\mathbf{( m m o l} / \mathbf{L})$ & Plasma potassium level & $\begin{array}{l}\text { Potassium in blood gas } \\
\text { (mmol/L) }\end{array}$ & WBC count $\times \mathbf{~ 1 0}^{\mathbf{9} / \mathbf{L}}$ \\
\hline 1 & 7.5 & N/D & 3.9 & 400 \\
2 & 7.2 & N/D & N/D & N/D \\
3 & 6.3 & 7.4 & N/D & 440 \\
$4^{*}$ & 6.8 & N/D & 2.5 & 360.51 \\
5 & 8.4 & N/D & 3.1 & N/D \\
6 & 6.7 & N/D & 4.4 & 392.1 \\
$7^{* *}$ & 4.5 & N/D & 4.3 & 135 \\
\hline
\end{tabular}

$\mathrm{N} / \mathrm{D}$ : not done

*Patient received treatment for hyperkalemia

**24-h post-chemotherapy

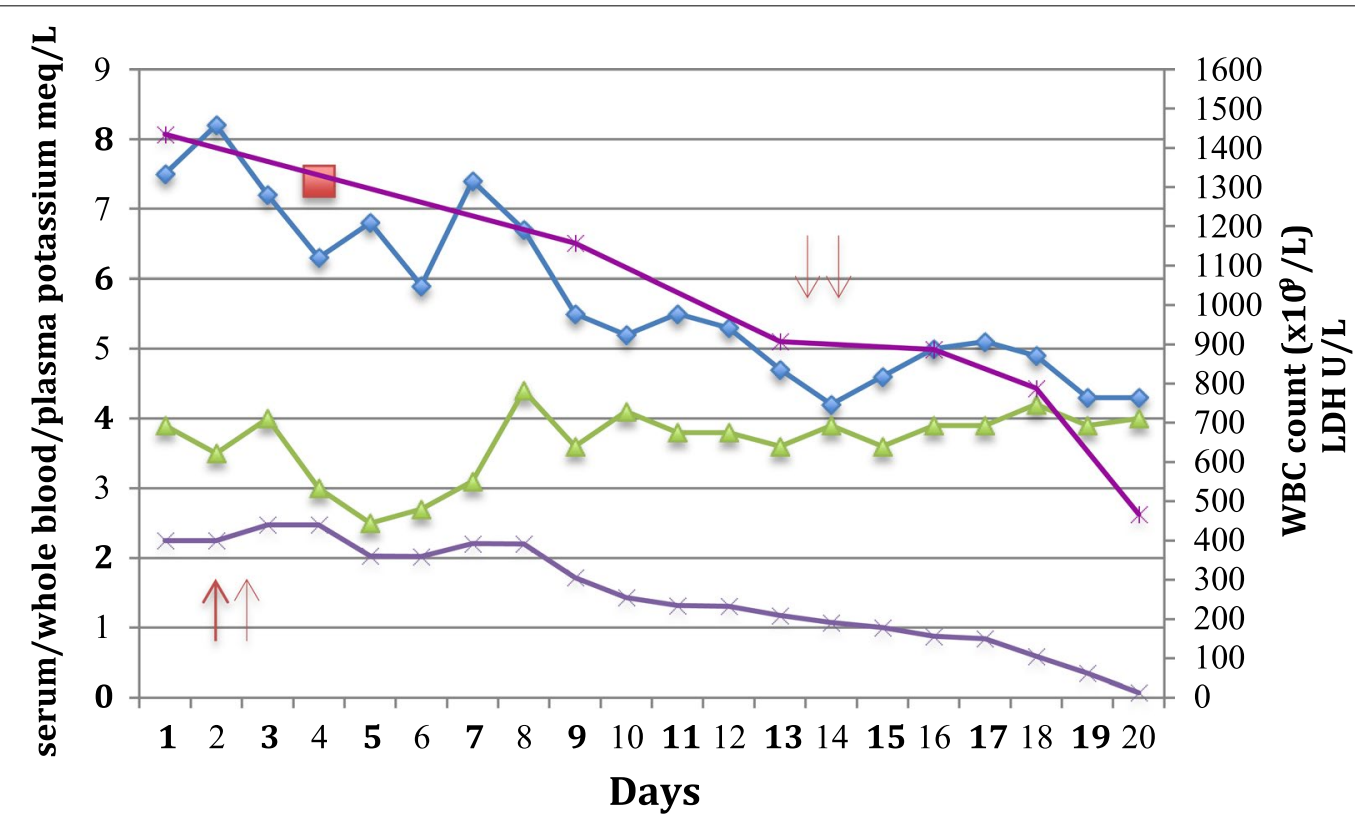

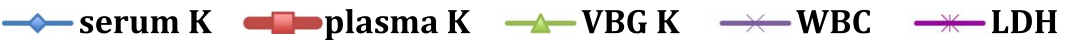

Fig. 1 Serum potassium, whole blood potassium levels and white blood cell counts in a patient with T-cell acute lymphocytic leukemia. Showed the discrepancy between serum (high level) compared to whole blood potassium level (low-normal), which indicates pseudohyperkalemia. Plasma potassium (red dot) is even higher than serum potassium, which indicates reverse pseudohyperkalemia. Potassium level in blood gas dropped to 2.5 after receiving K-lowering agent (up-arrow). Serum potassium level normalized when WBC count dropped below 150 (down-arrow)

potassium level $>7 \mathrm{mmol} / \mathrm{L}$ is potentially lethal due to its cardiotoxicity $[6,7]$.

Since the ratio of total body intracellular to extracellular potassium is approximately $40: 1$, while the ratio of circulating blood cells to plasma potassium is approximately 20-30:1, any minute release of intracellular potassium can inaccurately raise its serum or plasma levels. Consequently, it is essential to differentiate between true hyperkalemia and spurious PHK, in order to avoid exposing the patient to unnecessary medications and needless acute dialysis that carries significant adverse effects and can lead to fatal hypokalemia [7, 8] Unfortunately, in the case described above, this was recognized following the patient having received medical treatment for hyperkalemia, where venous blood-gas potassium level dropped significantly, though without complications (Table 3, Fig. 1).

Spurious hyperkalemia is considered when in vitromeasured serum (or plasma) potassium level is falsely raised to above the local reference range upper limit, 


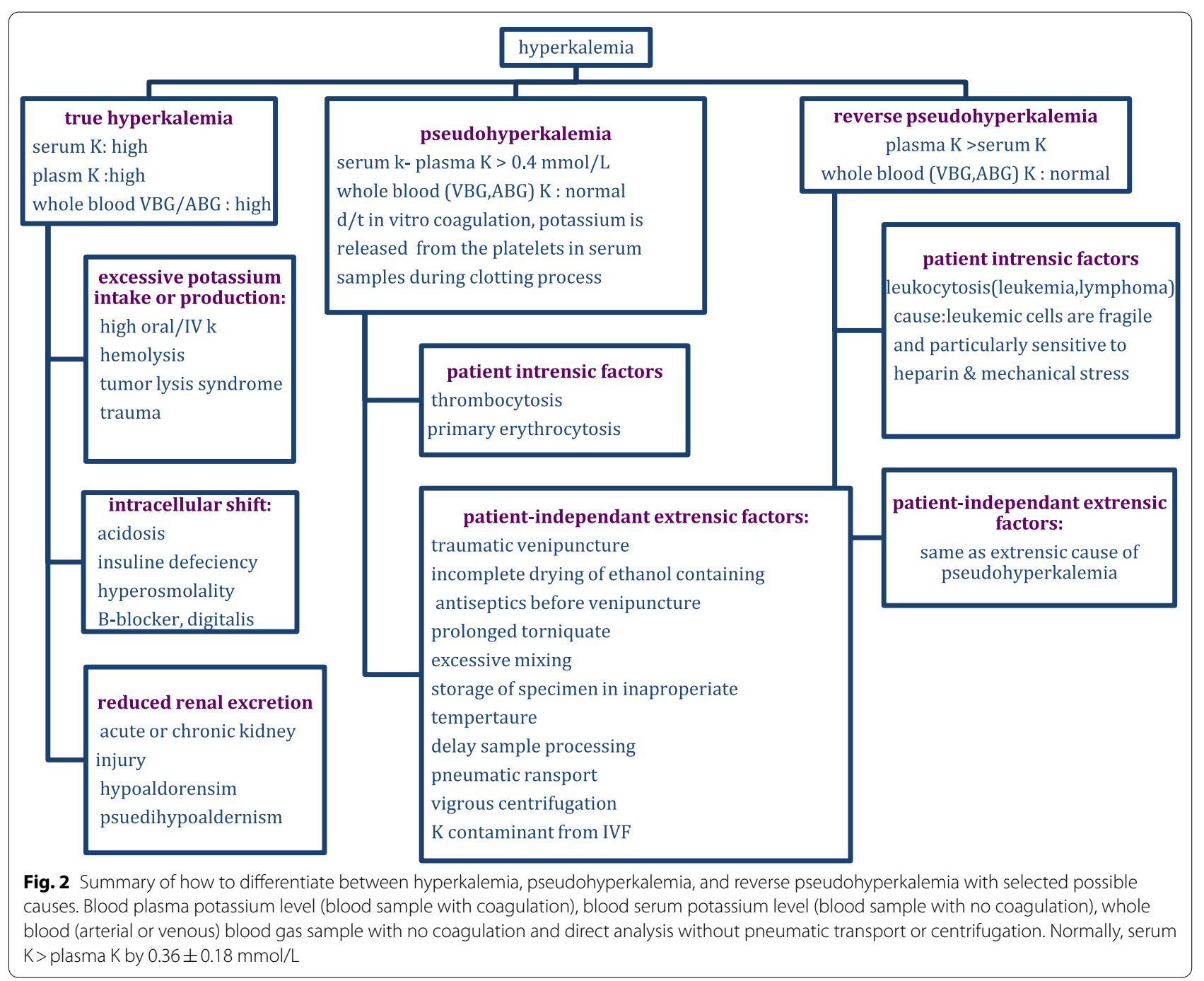

while the actual in vivo level is normal [4]. There are two defined clinical conditions that lead to spurious hyperkalemia: PHK and rPHK. Serum is defined as the remainder-portion of blood, post-coagulation. However, plasma is obtained when blood clotting is prevented, with addition of anti-coagulants such as heparin. Based on previous reports, PHK is defined as a serum potassium level exceeding plasma potassium level by $0.4 \mathrm{mmol} / \mathrm{L}$, provided that samples are collected under strict standardized techniques, maintained at room temperature $\left(15-25{ }^{\circ} \mathrm{C}\right)$, and analyzed within one hour post-blood sample collection $[7,8]$.

PHK was first described by Hartmann and colleagues in 1955, where he reported a case of hyperkalemia in a patient with thrombocytosis and attributed this to the excess of potassium released by platelets during the clotting process $[2,3]$. Thereafter, several theories were postulated for the possible etiology of PHK, including in vitro hemolysis during blood sample collection using narrow-gauge needles, fist clenching during phlebotomy and prolonged use of a tourniquet. Other researchers suggested that in vitro hemolysis could result from improper processing of blood samples, such as vigorous shaking of samples postcollection, prolonged sample incubation period in inappropriate temperatures, and excessive centrifugation [7, 9-11],(Fig. 2). Ku and colleagues, similarly to Dickinson and colleagues, had compared plasma and serum potassium levels in acute leukemia using two transport methods, concluding that the potassium level is highly exacerbated within pneumatic transport of samples, in comparison to messenger / hand transport [7, 12]. These possible extrinsic causes of PHK might be exaggerated in patients with extreme hyperleukocytosis, thrombocytosis, and polycythemia, especially in cases of leukemia-where the cell fragility is highly increased. 
(Table 3, Fig. 2). Moreover, inherited defects in the erythrocyte membrane structure that causes in vitro leak of potassium, such as leaky cell syndrome (familial pseudohyperkalemia) were reported in selected cases [7, 9-11](Fig. 2).

Our patient initially demonstrated an elevated serum potassium level. Consequently, in order to eliminate all possible mechanical causes of cell lysis-secondary to delayed transportation or using a pneumatic transport system-blood samples were collected by an expert nurse from the central line and sent immediately to the hospital laboratory by hand for analysis. The analyzed serum potassium level was $6.3 \mathrm{mmol} / \mathrm{L}$, in comparison to the serum potassium level of $7.5 \mathrm{mmol} / \mathrm{L}$, from another sample transported through pneumatic tube facilities. This discrepancy indicated that pneumatic transport can play a role in the pseudo-elevation of serum potassium. This was also demonstrated by Garwicz et al., together with and Huang and colleagues, where both research groups identified that pneumatic tube-transported samples resulted in exacerbated plasma potassium levels in comparison to manually-transported samples in a patient with chronic lymphocytic leukemia (CLL) and hyperleukocytosis $[9,13]$.

In addition, our medical team considered the possibility of potassium discharge from platelets during the clotting process within the serum sample, so an additional plasma blood sample was collected within a heparinized tube and sent immediately to the laboratory by hand, along with another serum sample (un-heparinized tube). Surprisingly, the potassium level was found to be elevated within plasma, in comparison to the serum potassium level $(7.42 \mathrm{mmol} / \mathrm{L}$ and $6.4 \mathrm{mmol} / \mathrm{L}$, respectively). This raised the possibility of rPHK, where plasma-potassium levels are typically higher than serum- potassium levels, and is an opposing clinical finding in comparison to PHK.

Singh et al. first described rPHK in 1997, in a patient with chronic lymphocytic leukemia (CLL) when the team noticed a discrepancy in potassium levels (between heparinized and un-heparinized blood samples) [1]. Previous literature concerning $\mathrm{rPHK}$ is limited, although our literature review identified 14 case reports that were predominantly adult patients with chronic lymphocytic leukemia (CLL) and lymphoma [1, 9, 13-22]. This study identified only one case similar to the above-described case, that was reported in a child with ALL, indicating that $\mathrm{rPHK}$ can occur in any age group [23]. Interestingly, Mansour and colleagues hypothesized that rPHK might carry a good prognostic indicator for CLL patients, since rPHK has a directly-proportional correlation with higher fragile cell numbers (i.e. smudge cell in CLL) which is a known prognostic factor in CLL [24].
The mechanism of rPHK is not clearly understood, though several observations have been made. Meng and colleagues demonstrated that increasing heparin concentrations within collection tubes were associated with increased potassium and LDH levels, implying increased WBC lysis since no hemolysis was observed [15]. These findings are not surprising given that the cells in patients with leukemia are both fragile and in higher abundance, consequently having increased heparin sensitivity and susceptibility to lysis, particularly during processing, pneumatic-tube transportation, and centrifugation [25]. This could explain the finding in our leukemic patient, where her leukocyte count was extremely high (WBC $>400 \times 10^{9} / \mathrm{L}$, with $90 \%$ blasts). Furthermore, the clotting process within serum samples might prevent movement of entangled white blood cells and, consequently, minimize their exposure to traumatic lysis and leading to lower serum potassium levels, in comparison to plasma levels [26]. This theory was argued against by El Shamy and colleagues in their recent retrospective cohort study, who demonstrated that $44 \%$ of the study population had developed rPHK in the absence of leukocytosis, with approximately $17 \%$ of of this cohort having a hematological malignancy [20].

Another theory suggested that severe leukocytosis and malignant cells have increased consumption of metabolic fuels, causing depletion of adenosine triphosphate (ATP), that can lead to impaired $\mathrm{Na}+/ \mathrm{K}+$ ATPase pump activity (which typically maintains intracellular potassium levels constant). This results in the extracellular release of potassium from the exacerbated number of WBCs that are present in such cases $[9,14,15,17,27]$.

An alternative explanation could be that leukemic cells undergo in vitro lysis, which leads to the release of cytoplasmic adenosine triphosphate (ATP) into plasma. Extracellular ATP has previously been shown to increase the in vitro cation permeability of lymphocytes from patients with CLL [17]. Influx of monovalent sodium $(\mathrm{Na}+)$ and lithium $(\mathrm{Li}+)$ ions was increased, while there also was a decrease in total cellular potassium $(\mathrm{K}+)$ levels, suggesting that extracellular ATP is an energy source for the active transport of potassium ions out of undamaged cells [17].

Considering other contributing factors for the pseudoelevation of potassium levels in serum and plasma samples, blood gas or whole-blood potassium level appears to be more accurate and remains the analysis of choice, particularly since it is a rapid and reliable analytical procedure due to the short interval between the sampledrawing and actual analysis, as demonstrated in the above-described case. Lee and colleagues have reported this observation in four adult cases with CLL, where plasma potassium levels were exacerbated in comparison 
to potassium levels following whole-blood gas analysis [25].

A total of six out of the 14 reported previous literature cases with $\mathrm{rPHK}$ had received a potassium-lowering agent, together with dialysis that was initiated in three of such cases. Some patients developed hypokalemia following such interventions and required potassium supplements (Table 2). This raised the importance of carefully interpreting elevated potassium levels in children with leukemia and hyperleukocytosis, who typically have a normal renal function, and instead consider the possibility of PHK or rPHK. Additionally, we recommend using potassium levels in whole-blood gas samples as a reference for the diagnosis and management of true hyperkalemia in patients with acute leukemia and hyperleukocytosis, in order to avoid any treatment-related complications.

\section{Conclusion}

Early recognition and diagnoses of $\mathrm{rPHK}$ are vital in all patients with significant leukocytosis (from hematologic neoplasms) that present with hyperkalemia, in the absence of other clinical or EKG-based evidence of hyperkalemia, in order to avoid any harmful outcomes following any invasive intervention.

We conclude that the potential for PHK and rPHK exists in serum and plasma samples, within the setting of leukocytosis in a patient with hematological malignancy. In such cases, whole-blood analysis is more accurate.

Finally, cancer centers should establish a mechanism through which their information system flags results of high potassium levels, specifically in patients with leukocyte counts $>100,000 / \mathrm{mm}^{3}$ that raise the possibility of PHK and hence minimize medical errors and improper management, in order to ensure a better quality of care and enhance patient safety.

Further investigation is still needed to identify in greater precision any factors that are associated with rPHK, and to clearly understand the pathophysiologic mechanisms underlying cell lysis.

\section{Abbreviations \\ PHK: Pseudohyperkalemia; rPHK: Reverse pseudohyperkalemia; ECG: Electro- cardiography; WBC: White blood cell; PICU: Pediatric intensive care unit; CBC: Complete blood count; Hgb: Hemoglobin; LDH: Lactate dehydrogenase; CLL: Chronic lymphocytic leukemia.}

\section{Acknowledgements}

Not applicable.

\section{Authors' contributions}

MA; collected all patient data and wrote the case. HA; systematic literature review, manuscript editing, prepared all tables/figures/graphs/references, and submitted the article for publication as 'corresponding author'. MK; wrote the manuscript and reviewed literature. AF; reviewed and edited the manuscript. $\mathrm{AO}$; reviewed and edited the manuscript.

Funding

Not applicable.

Availability of data and materials

Data sharing not applicable to this article as no data sets were generated or analyzed during the current study.

\section{Declarations}

Ethics approval and consent to participate

Not applicable.

Consent for publication

Not applicable.

\section{Competing interests}

The authors declare that they have no competing interests.

\section{Author details}

${ }^{1}$ Department of Pediatrics, Division of Pediatric Nephrology, King Faisal University-College of Medicine, AlHasa, Kingdom of Saudi Arabia. ${ }^{2}$ Multiorgan Transplantation Center, Pediatric Nephrology and Kidney Transplantation Section, King Fahad Specialist Hospital, Dammam, Kingdom of Saudi Arabia. ${ }^{3}$ Department of Critical Care, Division of Pediatric Critical Care, King Fahad Specialist Hospital, Ammar Bin Thabit Street, Dammam 32253, Kingdom of Saudi Arabia.

Received: 1 November 2020 Accepted: 27 October 2021

Published online: 11 December 2021

\section{References}

1. Singh PJ, Zawada ET, Santella RN. A case of "reverse" pseudohyperkalemia. Miner Electrolyte Metab. 1997;23(1):58-61.

2. Robson WL, Kabani AM, Leung AK, Fick GH, McKenna Al. The relationship of the serum potassium to the platelet count in children. Clin Pediatr. 1992;31(2):123-5.

3. Hartmann RC, Auditore JV, Jackson DP. Studies on thrombocytosis. I. Hyperkalemia due to release of potassium from platelets during coagulation. J Clin Invest. 1958;37(5):699-707.

4. Myerson RM, Frumin AM. Hyperkalemia associated with the myeloproliferative disorder. Arch Intern Med. 1960;106:479-82.

5. Bronson WR, DeVita VT, Carbone PP, Cotlove E. Pseudohyperkalemia due to release of potassium from white blood cells during clotting. N Engl J Med. 1966;274(7):369-75.

6. Cummings BM, Macklin EA, Yager PH, Sharma A, Noviski N. Potassium abnormalities in a pediatric intensive care unit: frequency and severity. J Intensive Care Med. 2014;29(5):269-74.

7. Dickinson H, Webb NJ, Chaloner C, Wynn RF, Bonney DK. Pseudohyperkalaemia associated with leukaemic cell lysis during pneumatic tube transport of blood samples. Pediatr Nephrol. 2012;27(6):1029-31.

8. Merritt M, Kline H, Garimella S, Seigler R. Pseudohyperkalemia in a patient with T-cell acute lymphoblastic leukemia and hyperleukocytosis. J Pediatr Intensive Care. 2018;7(3):166-8.

9. Garwicz D, Karlman M. Early recognition of reverse pseudohyperkalemia in heparin plasma samples during leukemic hyperleukocytosis can prevent iatrogenic hypokalemia. Clin Biochem. 2012;45(18):1700-2.

10. Ingram RH, Seki M. Pseudohyperkalemia with thrombocytosis. N Engl J Med. 1962:267:895-900.

11. Schneider AJ. Some thoughts on normal, or standard, values in clinical medicine. Pediatrics. 1960;26:973-84.

12. Ku AS, Chen RH, Law RL. Pseudohyperkalaemia with acute leukaemia: association with pneumatic tube transport of blood specimens. Hong Kong Med J. 2014;20(2):158-60. 
13. Huang N, Bufalino S, Czerlanis C. Pneumatic tube-induced reverse pseudohyperkalemia in a patient with chronic lymphocytic leukemia. Fed Pract. 2016;33(Suppl 5):60S-S62.

14. Abraham B, Fakhar I,Tikaria A, Hocutt L, Marshall J, Swaminathan S, et al. Reverse pseudohyperkalemia in a leukemic patient. Clin Chem. 2008;54(2):449-51.

15. Meng QH, Krahn J. Reverse pseudohyperkalemia in heparin plasma samples from a patient with chronic lymphocytic leukemia. Clin Biochem. 2011;44(8-9):728-30

16. Avelar T. Reverse pseudohyperkalemia in a patient with chronic lymphocytic leukemia. Perm J. 2014;18(4):e150-2.

17. Mansoor S, Holtzman NG, Emadi A. Reverse pseudohyperkalemia: an important clinical entity in chronic lymphocytic leukemia. Case Rep Hematol. 2015;2015:930379.

18. Juan L-T. Reverse pseudohyperkalemia in a patient with acute lymphoblastic leukemia: a case report. Tungs' Med J. 2017;13:37-42.

19. Avelar F. A case of reverse pseudohyperkalemia in a patient with atypical chronic lymphocytic leukemia. Ann Hematol Oncol. 2020;7(9):1321.

20. Shamy OE. Reverse pseudohyperkalemia is more than leukocytosis: a retrospective study. Clin Kidney J. 2021:14(5):1443-9.

21. Ahmed YRM, Houston J, Al Jaber E. Reverse pseudohyperkalemia: Verify potassium levels before initiating treatment. Int J Case Rep Images. 2020.

22. Jafar MS, Thalambedu N, Kolandra L, Roomi S. False, reversed but not true: a curious case of hyperkalemia. Cureus. 2020;12(8):e10066.

23. Garwicz D, Karlman M, Øra I. Reverse pseudohyperkalemia in heparin plasma samples from a child with T cell acute lymphoblastic leukemia with hyperleukocytosis. Clin Chim Acta. 2011:412(3-4):396-7.

24. Moreno G, Gunsolus IL. Reverse pseudohyperkalemia and pseudohyponatremia in a patient with B-cell non-Hodgkin lymphoma. Clin Biochem. 2020;78:63-5.

25. Lee HK, Brough TJ, Curtis MB, Polito FA, Yeo KT. Pseudohyperkalemia-is serum or whole blood a better specimen type than plasma? Clin Chim Acta. 2008;396(1-2):95-6.

26. Dimeski G, Bird R. Hyperleukocytosis: pseudohyperkalaemia and other biochemical abnormalities in hyperleukocytosis. Clin Chem Lab Med. 2009;47(7):880-1.

27. Logan JG, Newland AC. Leucocyte sodium-potassium adenosine triphosphatase and leukemia. Clin Chim Acta. 1982;123(1-2):39-43.

\section{Publisher's Note}

Springer Nature remains neutral with regard to jurisdictional claims in published maps and institutional affiliations.

Ready to submit your research? Choose BMC and benefit from:

- fast, convenient online submission

- thorough peer review by experienced researchers in your field

- rapid publication on acceptance

- support for research data, including large and complex data types

- gold Open Access which fosters wider collaboration and increased citations

- maximum visibility for your research: over $100 \mathrm{M}$ website views per year

At BMC, research is always in progress.

Learn more biomedcentral.com/submissions 